Check for updates

Cite this: RSC Adv., 2017, 7, 33572

Received 13th March 2017 Accepted 19th June 2017

DOI: $10.1039 / c 7 r a 02994 b$

rsc.li/rsc-advances

\section{Curcumin re-sensitizes multidrug resistant (MDR) breast cancer to cisplatin through inducing autophagy by decreasing CCAT1 expression}

\author{
Lv Xiao-ai, (DD* Wang Bei, Xu Xiao-hong, Pan Lei, Wang Bin, Dong Xiao-xue, \\ Zheng Chen-hui and Du Qi-wei
}

\begin{abstract}
Multidrug resistance (MDR) is a major obstacle to the chemotherapeutic treatment of breast cancer. The aim of this study is to investigate the mechanisms of curcumin induced increased chemosensitivity in MDR breast cancer cells. The results of MTT and western blot showed that curcumin could sensitize MCF-7 and MCF-7/DDP cells to cisplatin and activate MCF-7/DDP cell autophagy. The levels of CCAT1, $\mathrm{p}-\mathrm{PI} 3 \mathrm{~K}, \mathrm{p}-\mathrm{AKT}$ and $\mathrm{p}-\mathrm{mTOR}$ detected by RT-PCR and western blot in MCF-7/DDP cells were higher than in MCF-7 cells, and curcumin downregulated their expression in a dose-dependent manner. Moreover, we found that downregulation of CCAT1 could re-sensitize MCF-7/DDP cells to cisplatin, inactivate the $\mathrm{PI3K/AKT/mTOR} \mathrm{pathway} \mathrm{and} \mathrm{activate} \mathrm{cell} \mathrm{autophagy,} \mathrm{while} \mathrm{overexpression} \mathrm{of} \mathrm{it} \mathrm{have} \mathrm{the} \mathrm{opposite}$ effect in MCF-7 cells. Inactivation of autophagy by 3-MA could reverse CCAT1 knockdown-induced increased chemosensitivity and inactivation of the PI3K/AKT/mTOR pathway in MCF-7/DDP cells. PLENCCAT1 could reverse curcumin-increased chemosensitivity and autophagy activation, however, inactivation of the PI3K/AKT/mTOR pathway by LY294002 could reverse once more pLEN-CCAT1decreased chemosensitivity and inactivation of autophagy. Finally, the xenotransplant nude mouse model was used to study the effect of CCAT1 on curcumin-induced autophagy in vivo, and the results showed that curcumin inhibited tumor volume growth, downregulated CCAT1 expression and induced autophagy in vivo. Curcumin decreased CCAT1 and inactivated the PI3K/Akt/mTOR pathway which could both activate autophagy, sensitizing MDR breast cancer cells to cisplatin.
\end{abstract}

\section{Introduction}

Breast cancer is the most common cancer and the second leading cause of cancer death in women worldwide. ${ }^{1}$ Drug resistance is the main barrier in the treatment of breast cancer. ${ }^{2}$ It is an urgent issue to effectively promote breast cancer chemosensitivity worldwide. Curcumin is a natural product derived from turmeric, and an anti-tumor agent via inducing cell cycle arrest at G2/M phases in various cancer cell lines. ${ }^{3}$ Additionally, curcumin could affect various transcription factors and protein kinases to prevent the transformation, proliferation, and invasion of tumor cells. The influence of curcumin on reversing multidrug resistance in breast cancer was suggested to be involved in downregulating p-glycoprotein and inhibition of ATP activity. ${ }^{4}$ Identifying the underlying mechanisms of curcumin activity is therefore of major importance in the clinical application of chemotherapy.

Autophagy is a cellular process that involves self-degradation and recycling of macromolecules and cellular organelles. ${ }^{5}$

Department of Breast Surgery, The First Affiliated Hospital of Zhejiang Chinese Medicine University, 54 Post Road, Shangcheng, Hangzhou, Zhejiang 310006, China. E-mail: lvxiaoai021@163.com; Tel: +86-0571-87070921
Similar to the situation in normal cells, autophagy is critical for tumor cells to survive stressful conditions, and thus it has been implicated in tumor resistance to chemotherapy. ${ }^{6}$ Nevertheless, enhanced autophagy was usually observed in advanced stages of tumorigenesis, and some studies have reported that inhibition of autophagy could increase cancer chemo-sensitivity to cytotoxic drugs. Consequently, autophagy was considered a doubleedged sword in cancer development, but autophagy modulation will become a novel strategy to overcome cancer drug resistance. $^{7}$

The PI3K/Akt/mTOR pathway is a prototypic survival pathway that is constitutively activated in many types of cancer. ${ }^{8}$ It is reported that $\mathrm{PI} 3 \mathrm{~K} / \mathrm{AKT} / \mathrm{mTOR}$ pathway play a key role in breast cancer progression, drug resistance, and treatment, ${ }^{9}$ and inhibition of this pathway induced autophagy ${ }^{7}$ and re-sensitized MCF7 breast cancer cell line to drug chemotherapy. ${ }^{\mathbf{1 0}}$

Long noncoding RNAs (lncRNAs, >200 nucleotides) have recently emerged as important regulators in governing fundamental biological processes, and many of which play an important roles in tumorigenesis. ${ }^{\mathbf{1 1}}$ Colon-cancer-associated transcript-1 (CCAT1) is firstly discovered and overexpressed in colon cancer. Accumulating evidence reveals that CCAT1 is upregulated in various cancer tissues compared with adjacent 
normal tissues. ${ }^{\mathbf{1 2 - 1 4}}$ However, the expression of CCAT1 and its functional mechanisms in multidrug resistant breast cancer are still unclear.

The results form our study showed that activation of autophagy could sensitize MDR breast cancer cells to cisplatin via a mechanism associated with curcumin-induced downregulated CCAT1 and inactivated PI3K/Akt/mTOR pathway.

\section{Materials and methods}

\subsection{Cell lines and drug treatment}

The human breast cancer cell line MCF-7 was purchased from Tumor Cell Bank of the Chinese Academy of Medical Science (Shanghai, China). MCF-7/DDP cells were derived by treating MCF-7 cells with cisplatin. The two cell lines were cultivated in RPMI-1640 supplemented with 10\% fetal bovine serum and 1\% penicillin and streptomycin at $37{ }^{\circ} \mathrm{C}$ in a humidified incubator with $5 \% \mathrm{CO}_{2}$. Drug treatment: the cells were treated with curcumin $(0,5,10,20,50 \mu \mathrm{M})$ for $24 \mathrm{~h}$ or with $20 \mu \mathrm{M}$ curcumin for different times $(0,12,24,36 \mathrm{~h})$, meanwhile, incubated with cisplatin $(1 \mu \mathrm{M}$ or $6 \mu \mathrm{M})$ for $48 \mathrm{~h}$. Additionally, 3-MA (autophagy inhibitors) with $5 \mathrm{mM}$ was used to inhibited autophagy, and LY294002 (PI3K-Akt-mTOR inhibitor) with $50 \mu \mathrm{M}$ was used to inhibited PI3K-Akt-mTOR pathway in MCF-7/DDP and MCF-7 cells, respectively.

Construction of stable cell lines with overexpression and downregulation of CCAT1 in MCF-7 and MCF-7/DDP cell, respectively.

Breast cancer cell lines stably expressing CCAT1, MCF-7 cells were transfected with the plasmid pLENT-CCAT1, and screened with puromycin $\left(2 \mu \mathrm{g} \mathrm{m} \mathrm{m}^{-1}\right)$ for four weeks. Cell lines stably suppressing CCAT1 were constructed by transfection with a lentivirus construct containing the desired vector (si-CCAT1), and screened with puromycin $\left(2 \mu \mathrm{g} \mathrm{ml}^{-1}\right)$ for four weeks.

\subsection{In vitro chemo-sensitivity assay}

Chemo-sensitivity was measured by MTT assay (Sigma, USA). Cells cultured in 96-well plates were treated with drugs for $48 \mathrm{~h}$, and the MTT solution ( $5 \mathrm{mg} \mathrm{ml}^{-1}, 20 \mu \mathrm{l}$ ) was added to each well. After incubation for $4 \mathrm{~h}$, the media was removed and DMSO (100 $\mu l)$ were added into each well. The relative number of surviving cells was assessed by measuring the OD value at $560 \mathrm{~nm}$. All assays should be performed in triplicate.

\subsection{Real-time quantitative reverse-transcription polymerase chain reaction (qRT-PCR)}

The total RNA was isolated from tissues or cells using Trizol reagent (Invitrogen, USA). To detect the expression of CCAT1, reverse transcription was performed with Prime Script RT reagent Kit (Takara, Japan). RT-qPCR was performed with SYBR Prime Script RT-PCR Kits (Takara, Japan) according to the manufacturer's instructions. The level of CCAT1 was calculated using the $2^{-\Delta \Delta C_{\mathrm{t}}}$ method. Each sample was analyzed in triplicate.

\subsection{Western bolt analysis}

Cells were washed with PBS and centrifuged. And than cells were placed on ice for $30 \mathrm{~min}$ in RIPA buffer in the presence of a cocktail proteinase inhibitor (Sigma-Aldrich, USA). Quantified protein lysates were resolved in an SDS-PAGE gel and were transferred onto PVDF membrane (Millipore, USA). The membrane was blocked in 5\% bovine serum albumin for $2 \mathrm{~h}$, and incubated with the primary antibody. After incubation with the secondary antibody for $2 \mathrm{~h}$ at room temperature, the immunoreaction was visualized using the ECL Advance reagent (GE Healthcare) and was quantified using Quantity One software.

\subsection{Breast cancer xenografts}

MCF-7/DDP cells transfected with pLED-CCAT1 were resuspended in PBS at $5 \times 10^{7}$ cells per $\mathrm{ml}$ and were injected into mammary gland fat pad of nude mice. Tumors volume was measured with a caliper and calculated as the formula: volume $\left(\mathrm{mm}^{3}\right)=$ width $^{2} \times$ length/2. After the tumor size reached $5 \times 5$ $\mathrm{mm}^{3}$, curcumin and cisplatin were administered by intraperitoneal injection. The tumor tissues were removed at the end of the experiment and subjected to qRT-PCR or western blotting. All the animal studies involving animal experiments were reviewed and approved by the Ethics Committee of the First Affiliated Hospital of Zhejiang Chinese Medicine University. All experiments were performed in compliance with the Chinese laws.

\subsection{Statistical analysis}

The data were expressed as means \pm SD at least three independent experiments. SPSS 17.0 software was used for statistical analyses, and a Student's $t$-test was used to determine the significance of differences between two groups. $P<0.05$ was considered statistically significant.

\section{Results}

3.1. Curcumin reduced cisplatin resistance and induced cell autophagy in breast cancer cells

Platinum compounds are the key components of chemotherapy for the treatment of breast cancer patients. Tumors are resistant to cisplatin-induced cytotoxicity, and therefore represent a significant obstacle to successful treatment outcome. Chemoresistance has been a major obstacle in the clinical treatment of breast cancer. To determine whether curcumin have influence on cisplatin resistance and cell autophagy in breast cancer cells, we first examined the chemo-sensitivity and autophagy of MCF7 and MCF-7/DDP cells treated with curcumin and cisplatin. As shown in Fig. 1A, the relative cell viability were both decreased with increasing concentration of curcumin in MCF-7 and MCF7/DDP cells. Also, the MCF-7 and MCF-7/DDP cells were incubated with $20 \mu \mathrm{M}$ curcumin, meanwhile, treated with $1 \mu \mathrm{M}$ and $6 \mu \mathrm{M}$ cisplatin, respectively. The relative cell viability were both decreased with increase of curcumin treatment time (Fig. 1B). The cell autophagy was performed with LC3-I, LC3-II and beclin-1 protein expression by western blot. As Fig. 1C and D showed that, LC3-I expression was decreased with increasing 
A

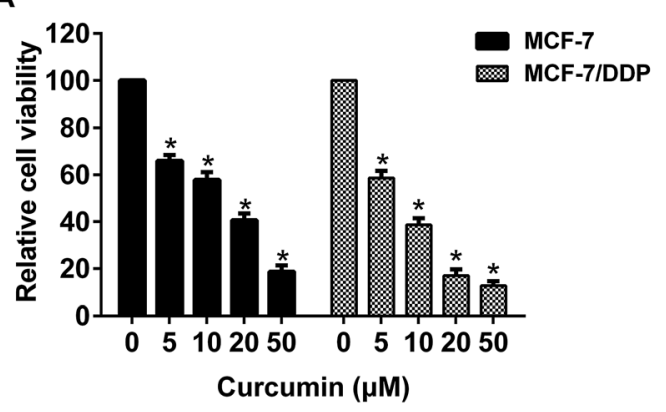

C

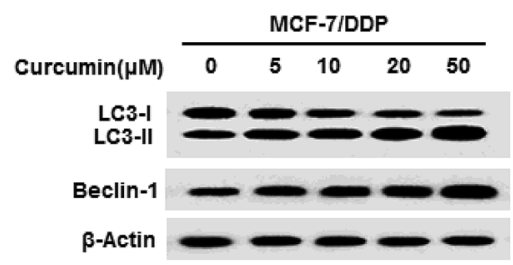

B
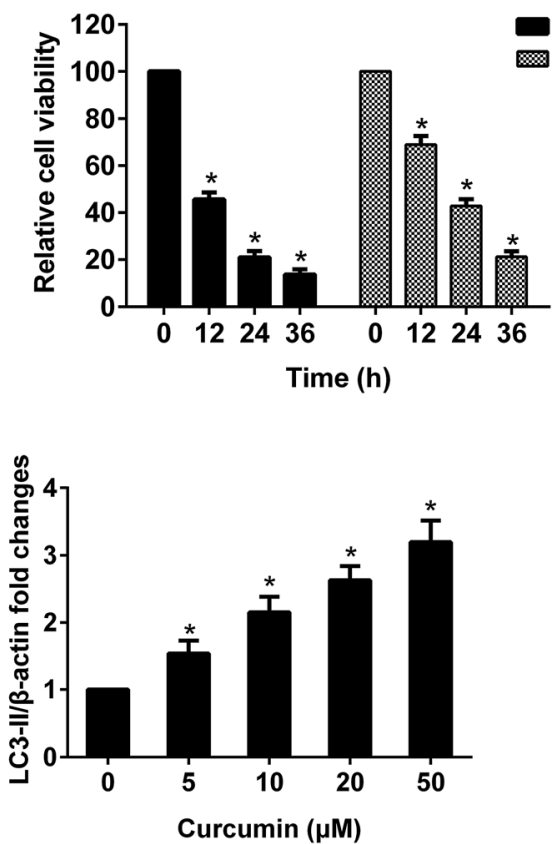

Fig. 1 The effect of curcumin on cisplatin resistance and cell autophagy in breast cancer cells. (A) The relative cell viability was decreased with increasing concentration of curcumin. (B) The relative cell viability was decreased with increase of curcumin treatment time. (C) LC3-I expression was decreased with increasing concentration of curcumin, but LC3-II and beclin-1 expression was opposite which suggesting that curcumin could induce MCF-7/DDP cell autophagy. $* P<0.01$ as compared with control.

A

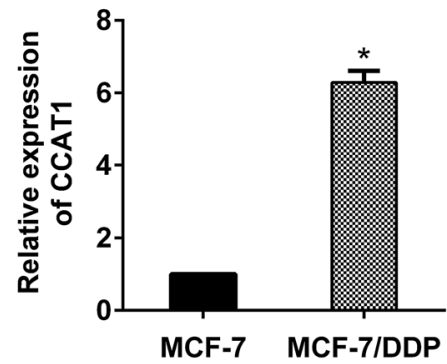

C

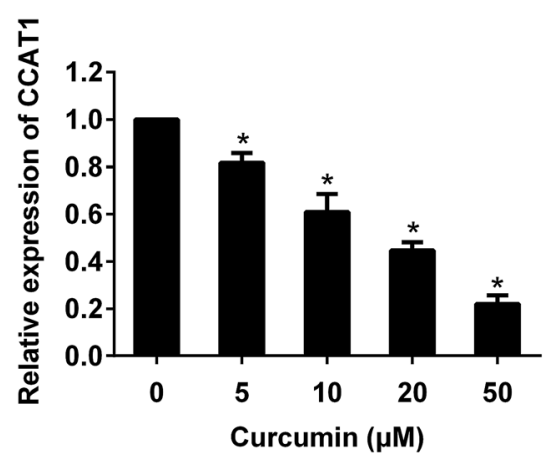

B

MCF-7 MCF-7/DDP

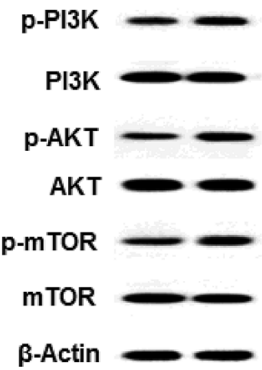

D

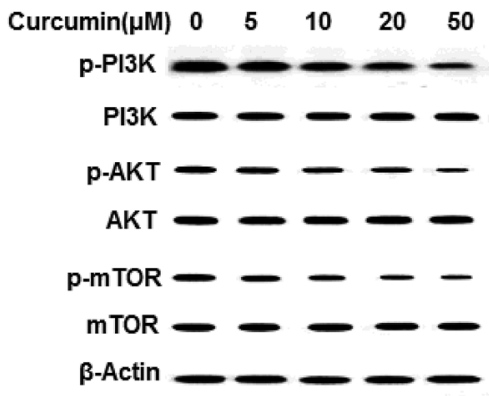

Fig. 2 The effect of curcumin on CCAT1 expression and PI3K-Akt-mTOR pathway in breast cancer cells. (A) The CCAT1 expression was higher in MCF-7/DDP cells than that in MCF-7 cells. (B) The expressions of $p$-PI3K, $p$-Akt and p-mTOR were higher in MCF-7/DDP cells than that in MCF-7 cells. (C) The CCAT1 expression were decreased in a dose-dependent manner. (D) The levels of $p$-PI3K, $p$-Akt and p-mTOR were decreased in a dose-dependent manner. ${ }^{*} P<0.01$ as compared with control. 
concentration of curcumin, but LC3-II and beclin-1 expression were increased with increasing concentration of curcumin, which suggested that curcumin could induce MCF-7/DDP cell autophagy.

\subsection{Curcumin inhibited CCAT1 expression and PI3K-Akt- mTOR pathway}

We have found that CCAT1 expression was significant increased, and the PI3K-Akt-mTOR pathway was activated in MCF-7/DDP cell (Fig. 2A and B). To investigate the impact of curcumin on CCAT1 expression and PI3K-Akt-mTOR pathway, the MCF-7/DDP cell were treated with $0,5,10,20$ and $50 \mu \mathrm{M}$ curcumin for $24 \mathrm{~h}$. The protein expression of important signaling molecules such as PI3K, Akt, and mTOR were determined by western blot. As Fig. 2C and $\mathrm{D}$ showed that, curcumin dramatically reduced $\mathrm{p}-\mathrm{PI} 3 \mathrm{~K}, \mathrm{p}-$ Akt, and p-mTOR expression in a dose-response manner whereas the total protein levels of PI3K, Akt, and mTOR remained unchanged. Thus, curcumin could inhibit CCAT1 expression and PI3K-Akt-mTOR pathway activation.

\subsection{CCAT1 expression influenced drug resistance and cell autophagy in breast cancer cells}

We have known that CCAT1 expression was significant increased in MCF-7/DDP cell. To determine whether CCAT1 is involved in the molecular etiology of chemo-resistance in breast cancer cells, we detected the biological functions of CCAT1 in breast cancer cells against cisplatin. The MCF-7 and MCF-7/DDP cells were stably transfected with CCAT1 expression vector PLEN-CCAT1 or CCAT1-specific siRNA, respectively, using the empty vector or control siRNA as a negative control (NC). We have detected the effect of CCAT1 expression on drug resistance, cell autophagy and PI3K-Akt-mTOR pathway. As Fig. 3A and B showed, the chemosensitivity of MCF-7/DDP cell transfected with si-CCAT1 was significantly increased, however, the chemosensitivity of MCF-7 cell transfected with pLEN-CCAT1 was higher than that transfected with pLEN-NC, suggesting that upregulated CCAT1 could promote drug resistance in breast cancer cells. As Fig. 3C and D showed, the cell autophagy of MCF-7/DDP cell transfected with si-CCAT1 was increased compared to that transfected with

B

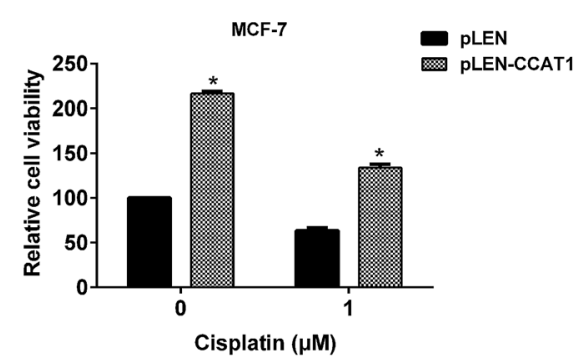

D
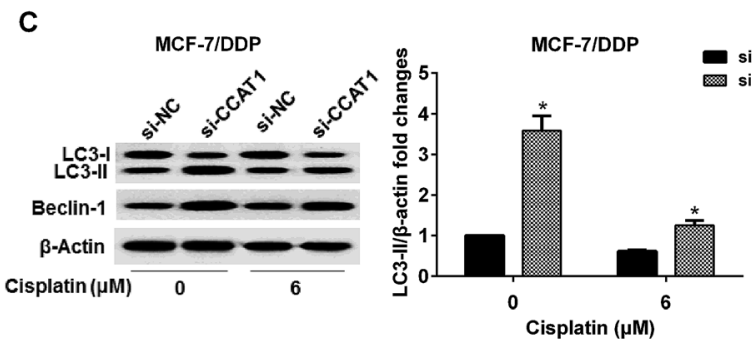

E

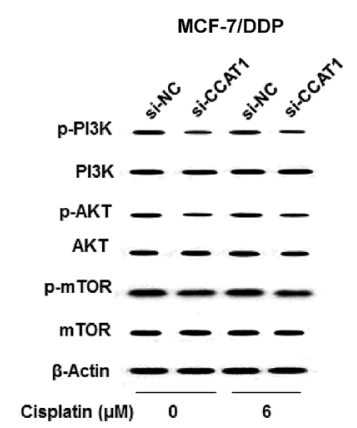

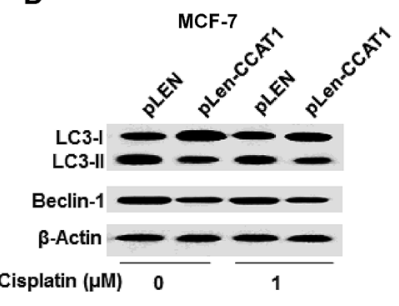

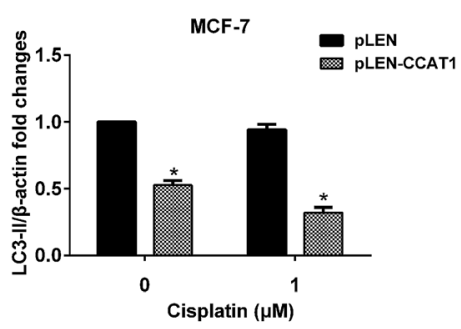

$\mathbf{F}$

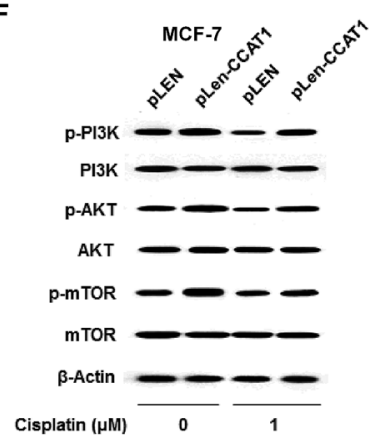

Fig. 3 The effect of CCAT1 on drug resistance and cell autophagy in breast cancer cells. (A) The cell viability of MCF-7/DDP cell transfected with si-CCAT1 was lower than that of control. (B) The cell viability of MCF-7 cell transfected with pLEN-CCAT1 was higher than that of control. (C) The cell autophagy of MCF-7/DDP cell transfected with si-CCAT1 was increased than that of control. (D) The cell autophagy of MCF-7 cell transfected with pLEN-CCAT1 than that transfected of control. (E) The PI3K-AKT-mTOR pathway were inhibited in MCF-7/DDP cell transfected with si-CCAT1 compared to control. (F) The PI3K-AKT-mTOR pathway were activated in MCF-7 cell transfected with pLEN-CCAT1 compared to control. ${ }^{*} P<0.01$ as compared with si-NC or pLEN-NC. 
A

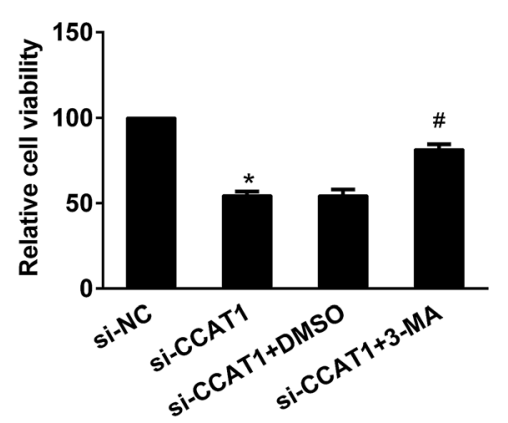

B

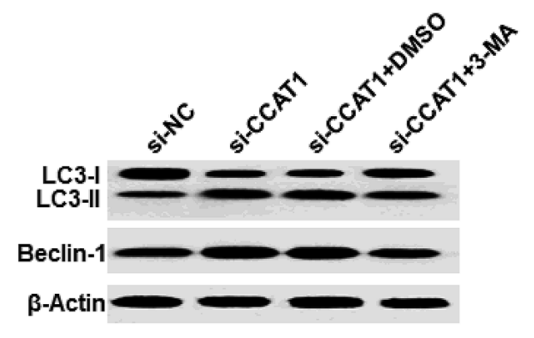

C

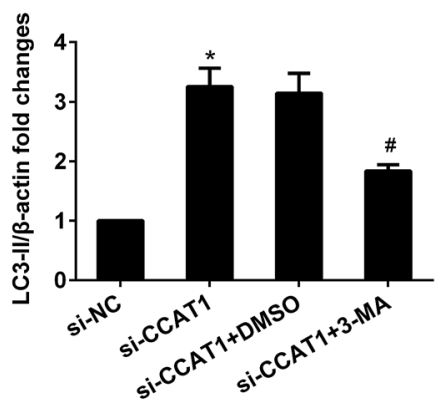

Fig. 4 The effect of CCAT1 knockdown on drug resistance and cell autophagy could been reversed by 3-MA (autophagy inhibitors) in MCF-7/ DDP cells. (A) Downregulation of CCAT1 increased chemosensitivity which could been reversed by 3-MA (autophagy inhibitors). (B) Downregulation of CCAT1 induced cell autophagy which could been reversed by 3-MA. (C) Downregulation of CCAT1 inhibited PI3K-AKT-mTOR pathway which could been reversed by $3-\mathrm{MA}$. $* P<0.01$ as compared with si-NC and \#P<0.01 as compared with si-CCAT1 + DMSO

si-NC, but the cell autophagy of MCF-7 cell transfected with pLEN-CCAT1 was decreased compared to that transfected with pLEN-NC, suggesting that upregulated CCAT1 could inhibit autophagy activity in breast cancer cells. As Fig. $3 \mathrm{E}$ and $\mathrm{F}$ showed, the PI3K-AKT-mTOR pathway were inhibited (reduced p-PI3K, pAkt, and p-mTOR expression) in MCF-7/DDP cell transfected with si-CCAT1, while the PI3K-AKT-mTOR pathway were activated (increased p-PI3K, p-Akt, and p-mTOR expression) in MCF-7 cell transfected with pLEN-CCAT1, suggesting that downregulated CCAT1 could inhibit PI3K-AKT-mTOR pathway activation in breast cancer cells.

3.4. The effect of CCAT1 knockdown and cell autophagy on drug resistance and PI3K/Akt/mTOR pathway in MCF-7/DDP cells

In order to evaluate the effect of cell autophagy on drug resistance under CCAT1 expression suppressed circumstances,
MCF-7/DDP cell transfected with si-CCAT1 was treated with/ without 3-MA (autophagy inhibitors). According to our results that, downregulation of CCAT1 increased chemosensitivity which could been reversed by 3-MA (Fig. 4A). Downregulation of CCAT1 induced cell autophagy that could been reversed by 3MA (Fig. 4B). In addition, the effect of CCAT1 knockdown and autophagy inhibition on PI3K/Akt/mTOR pathway in MCF-7/ DDP cells was analyzed by western blot. As shown in Fig. 4C, decreased CCAT1 downregulated p-PI3K, p-Akt, and p-mTOR expression which were reversed by 3-MA. Therefor, inactivation of autophagy could desensitize MDR breast cancer cells to cisplatin and activated PI3K/Akt/mTOR pathway.

\subsection{The effect of CCAT1 and PI3K-AKT-mTOR pathway on curcumin-induced cell autophagy in MCF-7/DDP cells}

In order to evaluate the role of PI3K-AKT-mTOR pathway in drug resistance under CCAT1 highly expression circumstances, MCF-
A

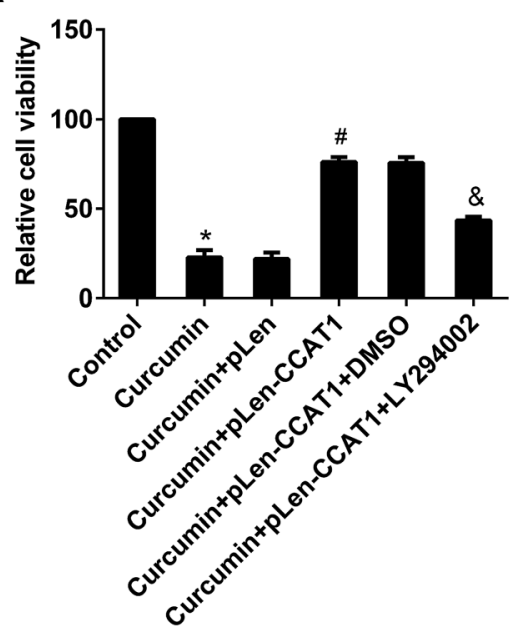

B

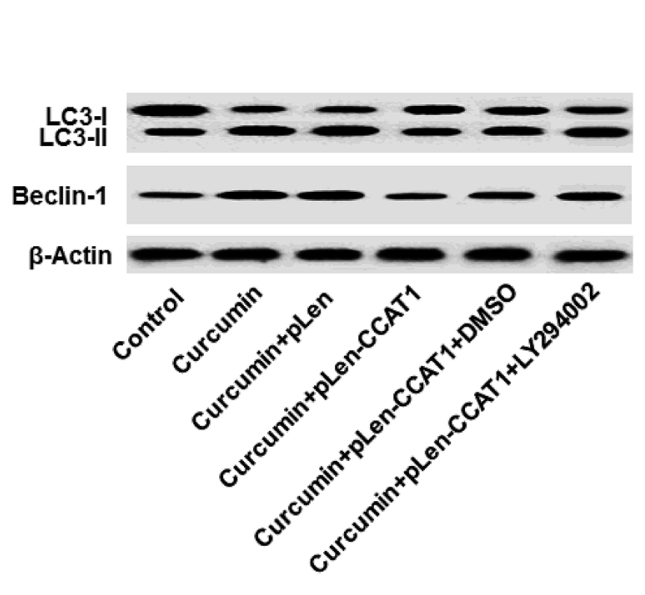

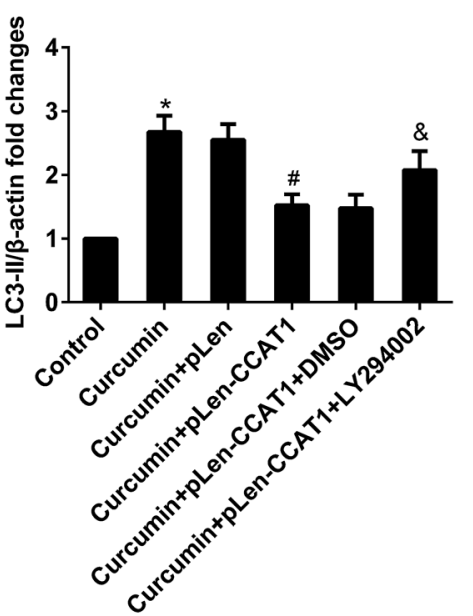

Fig. 5 The effect of CCAT1 and PI3K-AKT-mTOR pathway on curcumin-induced cell autophagy in MCF-7/DDP cells. (A) Curcumin-increased chemosensitivity was reversed by pLEN-CCAT1, which was reversed once more by LY294002 (PI3K-Akt-mTOR inhibitor). (B) Curcumin-induced cell autophagy was reversed by pLEN-CCAT1, which was reversed once more by LY294002 (PI3K-Akt-mTOR inhibitor). *P<0.01 as compared with control, $\# P<0.01$ as compared with curcumin + pLEN-NC, and $\& P<0.01$ as compared with curcumin + pLEN-CCAT1 + DMSO. 
7/DDP cells were treated curcumin transfected with/without pLEN-CCAT1 and/or LY294002. As shown in Fig. 5A, curcumin-increased chemosensitivity was reversed by pLENCCAT1, which was reversed once more by LY294002 in MCF-7 cells. Curcumin-induced cell autophagy was reversed by pLEN-CCAT1, which was reversed once more by LY294002 (Fig. 5B). Thus, curcumin, decreased CCAT1 expression and inhibition of PI3K-Akt-mTOR were potential treatments to sensitize MDR breast cancer cells to cisplatin.

\subsection{Overexpression of CCAT1 increased tumor growth and decreased curcumin-induced autophagy in xenotransplant nude mouse model}

To investigate whether CCAT1 enhanced resistant of breast tumor to cisplatin in vivo, MCF-7/DDP cells transfected with pLEN-CCAT1 or pLEN-NC were injected subcutaneously into nude mice. After tumor size was up to $5 \times 5 \mathrm{~mm}^{3}$, and then they were given intraperitoneal injection of cisplatin and curcumin. As shown in Fig. 6A, curcumin could inhibit tumor volume growth compared to control. The tumors originating from cells expressing CCAT1 were bigger in size than those tumors originating from control cells combination treatment with curcumin. These results suggested that overexpression of CCAT1 promoted tumor growth. In addition, we found that curcumin-downregulated CCAT1 expression was reversed by pLEN-CCAT1 in vivo, which was consistent with the result in vitro (Fig. 6B). Moreover, curcumin could induce autophagy in xenotransplant nude mouse model, which was reversed by overexpression of CCAT1 (Fig. 6C). Thus, the downregulated CCAT1 expression that induced by curcumin could activate autophagy to sensitize MDR breast cancer to cisplatin.

\section{Discussion}

Breast cancer is currently the most lethal gynecologic malignancy in many countries, and the majority of patients with breast cancer ultimately succumb to recurrent, chemo-resistant disease. ${ }^{15}$ Several factors correlate with prognosis, ${ }^{16}$ however, a complete mechanism has not been defined to explain the development of chemo-resistance and progression of breast cancer. Autophagy is an evolutionarily conserved lysosomal degradation pathway critical for maintaining cellular integrity. ${ }^{\mathbf{1 7}}$ In cancer, autophagy have two opposing roles. On one hand, autophagy could suppress tumorigenesis. On the other hand, autophagy could serve as a pro-survival mechanism when
A

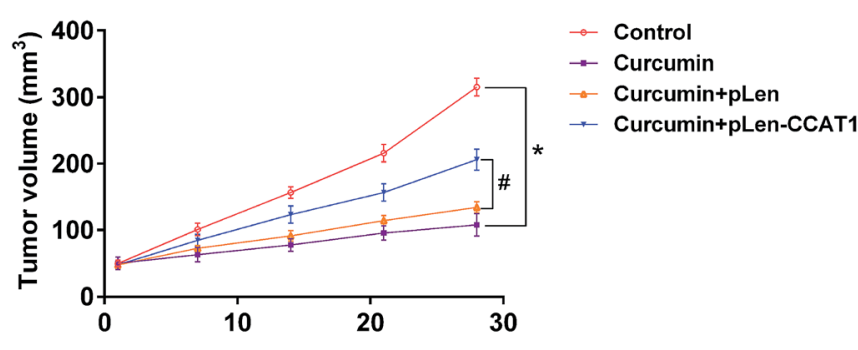

C

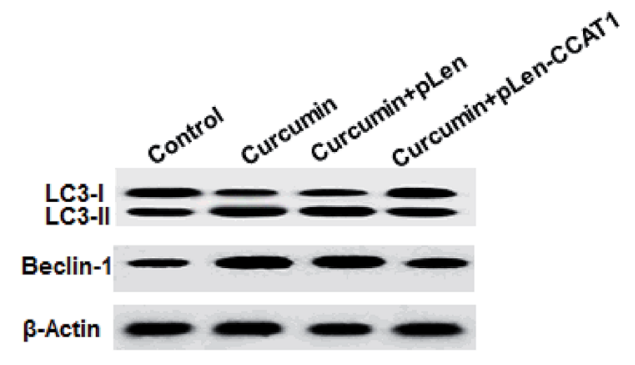

B

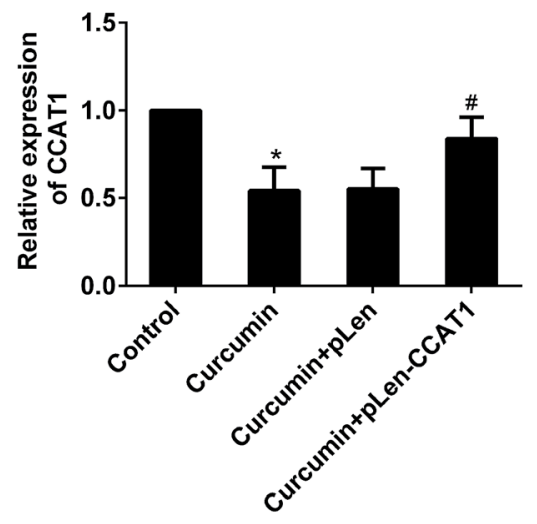

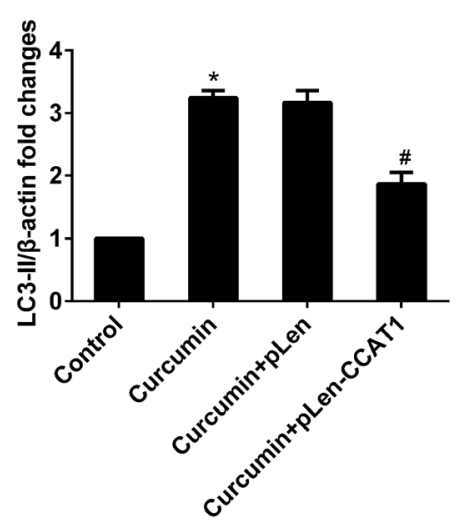

Fig. 6 The effect of pLED-CCAT1 on curcumin-induced autophagy in xeno-transplant nude mouse model. To establish a nude mice transplantation tumor model with MCF-7/DDP cells transfected with pLEN-CCAT1 or pLEN-NC, after tumor size was up to $5 \times 5$ mm ${ }^{3}$, and then they were given intraperitoneal injection of cisplatin and curcumin. (A) Curcumin-inhibited tumor volume growth was reversed by pLEN-CCAT1. (B) Curcumin-induced downregulated CCAT1 expression was reversed by pLEN-CCAT1. (C) Curcumin-induced autophagy was reversed by pLENCCAT1. $* P<0.01$ as compared with control and $\# P<0.01$ as compared with curcumin + pLEN-NC. 
cancer cells are subjected to damage by chemical or physical treatment. ${ }^{18}$ Phytochemicals derived from dietary sources can interfere with tumor promotion and progression, and generally have many molecular targets. This pleiotropism may constitute an advantage in the treatment of chemo-resistant breast cancer. The cytotoxic effects of curcumin have been reported in several tumor types. Curcumin has been shown to inhibit multi-drug resistant in lung cancer and ovarian cancer when combined with doxorubicin and paclitaxel, respectively. ${ }^{19,20}$ In this study, we found that curcumin could reduce cisplatin resistance though inducing cell autophagy in breast cancer cells. This results suggested that autophagy was a therapeutic target in MDR breast cancer.

The PI3K/Akt/mTOR pathway is constitutively activated in MDR cancer, ${ }^{21}$ and play a key role in progression, drug resistance of breast cancer. ${ }^{9}$ This study showed that the expressions of p-PI3K, p-Akt and p-mTOR were higher in MCF-7/DDP cells than that in MCF-7 cells. Moreover, the PI3K/Akt signaling pathway is upstream of the mammalian target of rapamycin (mTOR), which is important in the induction of autophagy. ${ }^{22}$ Curcumin has been found to interfere with PI3K/Akt signaling pathway leading to suppression of cell proliferation, invasion, and migration in various cancer cells. ${ }^{23-25}$ This study showed that the MCF-7/DDP cells were treated with different concentrations of curcumin, and the levels of p-PI3K, p-Akt and pmTOR were decreased in a dose-dependent manner. In addition, curcumin-increased chemosensitivity and inactivated autophagy were both reversed by LY294002. Theses results indicated that activation of autophagy could sensitize MDR breast cancer cells to cisplatin via a mechanism associated with curcumin-induced inactivated PI3K/Akt/mTOR pathway.

Non-coding RNAs have been shown to promote tumorigenesis, and the dysregulation of lncRNAs has also been shown to contribute to cancer pathogenesis, providing novel therapeutic opportunities to treat cancer. Long non-coding RNA CCAT1 is a novel biomarker of poor prognosis in patients with breast cancer, ${ }^{26}$ and acts as an oncogene and promotes chemoresistance in docetaxel-resistant lung adenocarcinoma cells. ${ }^{27}$ In this study, the CCAT1 expression was higher in MCF-7/DDP cells than that in MCF-7 cells, and could been suppressed by curcumin. Downregulation of CCAT1 inhibited drug resistance and cell autophagy in MCF-7/DDP cells, but CCAT1 knockdown have opposite results in MCF-7 cells. In addition, the effect of downregulation CCAT1 on drug resistance and cell autophagy could been reversed by 3-MA in MCF-7/DDP cells. These results suggested that activation of autophagy could sensitize MDR breast cancer cells to cisplatin via a mechanism associated with curcumin-induced downregulated CCAT1.

Knockdown of CCAT1 increased the chemo-sensitivity and autophagy of cisplatin-resistant MCF-7 cells and inactivated PI3K/Akt/mTOR pathway, whereas overexpression of CCAT1 decreased them. Furthermore, overexpression of CCAT1 reversed curcumin-increased chemosensitivity and autophagy inactivation, which could reversed once more by $\mathrm{LY} 294002 . \mathrm{Lu}^{28}$ et al. reported that miR-218 was negatively regulated by CCAT1 in HBE cells, and Zhang et al. ${ }^{29}$ have found that miR-218 suppressed colon cancer cells by targeting PI3KC2A, a key gene of the PI3K/Akt/mTOR signaling pathway. Whether the low levels of CCAT1 inactivated PI3K/Akt/mTOR pathway through targeting miR-218 might need a further experiment in the MDR breast cancer.

In summary, our data revealed that CCAT1 was a proresistant IncRNA that could regulate chemo-sensitive and autophagy in MDR breast cancer cells. Overexpression of CCAT1 decreased the sensitivity of MDR cells to cisplatin and inhibits autophagy in breast cancer cells. However, downregulation of CCAT1 increased the sensitivity of MDR cells to cisplatin and promoted autophagy in MDR breast cancer cells. Meanwhile, curcumin-decreased CCAT1 and inactivated PI3K/Akt/mTOR pathway could activate autophagy that could sensitize MDR breast cancer cells to cisplatin. Although further studies are needed to fully elucidate the definite regulatory mechanisms of CCAT1, this work suggests that decreased CCAT1 and inactivated PI3K/Akt/mTOR pathway induced by curcumin lead to activation of autophagy and which could sensitize MDR breast cancer cells to cisplatin. This study will provide new insight into the mechanisms underlying drug resistance, and curcumin can be an effective therapy to MDR breast cancer.

\section{Conflict of interest}

The authors declare no conflict of interest.

\section{Funding}

The work was supported by the Natural Science Foundation of Zhejiang Province (LQ17H270004), and the project of Health and Family Planning Commission of Zhejiang Province (2016KYB217).

\section{References}

1 R. Murley, Lancet, 2016, 20, 193.

2 T. Yuan, W. Yue, M. F. Kiani and B. Wang, Clin. Breast Cancer, 2016, 16, 335-343.

3 Y. Akkoç, Ö. Berrak, E. D. Arısan, P. Obakan, A. ÇokerGürkan and N. Palavan-Ünsal, Biomed. Pharmacother., 2015, 71, 161-171.

4 L. Lv, K. Qiu, X. Yu, C. Chen, F. Qin, Y. Shi, J. Ou, T. Zhang, H. Zhu, J. Wu, C. Liu and G. Li, J. Biomed. Nanotechnol., 2016, 12, 973-985.

5 N. Mizushima, Genes Dev., 2007, 21, 2861.

6 Q. Sun, T. Liu, Y. Yuan, Z. Guo, G. Xie, S. Du, X. Lin, Z. Xu, M. Liu and W. Wang, Int. J. Cancer, 2015, 136, 1003-1012.

7 X. Sui, R. Chen, Z. Wang, Z. Huang, N. Kong, M. Zhang, W. Han, F. Lou, J. Yang and Q. Zhang, Cell Death Dis., 2013, 4, e838.

8 J. Lopiccolo, G. M. Blumenthal, W. B. Bernstein and P. A. Dennis, Drug Resist. Updates, 2008, 11, 32-50.

9 A. Guerrerozotano, I. A. Mayer and C. L. Arteaga, Cancer Metastasis Rev., 2016, 1-10.

10 T. Komeilimovahhed, S. Fouladdel, E. Barzegar, S. Atashpour, M. H. Ghahremani, S. N. Ostad, Z. Madjd and E. Azizi, Iran. J. Basic Med. Sci., 2015, 18, 472-477. 
11 W. Peng, S. Si, Q. Zhang, C. Li, F. Zhao, F. Wang, J. Yu and R. Ma, J. Exp. Clin. Cancer Res., 2015, 34, 79.

$12 \mathrm{X}$. He, X. Tan, X. Wang, H. Jin, L. Liu, L. Ma, H. Yu and Z. Fan, Tumor Biol., 2014, 35, 12181-12188.

13 H. Zhu, X. Zhou, H. Chang, H. Li, F. Liu, C. Ma and J. Lu, Int. J. Clin. Exp. Pathol., 2015, 8, 5427.

14 M. Z. Ma, B. F. Chu, Y. Zhang, M. Z. Weng, Y. Y. Qin, W. Gong and Z. W. Quan, Cell Death Dis., 2015, 6, e1583.

15 J. Mao, B. Song, Y. Shi, B. Wang, S. Fan, X. Yu, J. Tang and L. Li, Int. J. Biochem. Cell Biol., 2013, 45, 1064-1073.

16 S. Das and T. S. Lin, Curr. Pharm. Des., 2017, 23(12), 18451859.

17 B. Levine and G. Kroemer, Cell, 2008, 132, 27-42.

18 M. M. Hippert, P. S. O'Toole and A. Thorburn, Cancer Res., 2006, 66, 9349-9351.

19 Y. Gu, J. Li, Y. Li, L. Song, D. Li, L. Peng, Y. Wan and S. Hua, Int. J. Nanomed., 2016, 11, 5757-5770.

20 Z. Liu, Y. Y. Zhu, Z. Y. Li and S. Q. Ning, Oncol. Lett., 2016, 12, 3944-3948.
21 H. Zhuang, J. Bai, J. Y. Chang, Z. Yuan and P. Wang, OncoTargets Ther., 2016, 7(51), 84688-84694.

22 A. Chen, L. J. Xiong, Y. Tong and M. Mao, Mol. Med. Rep., 2013, 8, 1011.

23 X. Xu, J. Qin and W. Liu, Gene, 2014, 546, 226-232.

24 W. C. Chen, Y. A. Lai, Y. C. Lin, J. W. Ma, L. F. Huang, N. S. Yang, C. T. Ho, S. C. Kuo and T. D. Way, J. Agric. Food Chem., 2013, 61, 11817-11824.

25 B. R. Seo, K. J. Min, I. J. Cho, S. C. Kim and T. K. Kwon, PLoS One, 2014, 9, e95588.

26 X. F. Zhang, T. Liu, Y. Li and S. Li, Int. J. Clin. Exp. Pathol., 2015, 8, 9440-9445.

27 J. Chen, K. Zhang, H. Song, R. Wang, X. Chu and L. Chen, OncoTargets Ther., 2016, 7, 62474-62489.

28 L. Lu, X. Hui, L. Fei, X. Liu, X. Lu, Q. Yang, J. Xue, C. Chao, S. Le and Q. Liu, Toxicol. Appl. Pharmacol., 2016, 304, 30.

29 X. Zhang, H. Shi, H. Tang, Z. Fang, J. Wang and S. Cui, Int. J. Mol. Med., 2015, 35, 1301. 